\title{
STABILITY SAFETY MARGIN BASED DESIGN OF LOW NOISE MICROWAVE AMPLIFIERS
}

\author{
KW Eccleston \\ Department of Electrical Engineering, National Universily of Singapore, Singapore 119260 \\ Email: elekwe@nus.edu.sg
}

\begin{abstract}
In low noise nicrowave armplifier design, particularly when using conditionally stable transistors, it is impossible to simultaneously achieve maximum gain, minimun noise figure and maximum port match. Hence it is necessary to trade-off these perforthance parameters which is usually accomplished with the aid of stability, gain, noise figure and mismatch (or VSWR circles described on the reflection coefficient planes. However, use of these graphical aids is often subjective, necessitating final optimisation using circuit design software. In this paper, we propose a different approach whereby the functional behaviour of two objective functions involving gain, noise figure and port mismatch are described as functions of the stability safety margin. This approach leads to an optimum design without requiring sophisticated optimisation algorithms and includes stability safety matgin as a design parameter.
\end{abstract}

\section{Introduction}

When designing low noise microwave amplifiers, it is the task to select suitable valtes for the soutce (input gencrator) and load reflection coefficicuts for the transistor. The source and load relection coefficients are chosen to achieve certain specifications for the amplifier performance parameters, such as gain, noise figure, port match, as wetl as ensure stability. Normally, it is impossible to simultaneously achieve maximum gain, minimum nojse figure and maximum port match; especially when using conditionally stable transistors. Hence a trade-off of these performance specifications is necessary [1].

The low noise amplifier design typically begins with the selection of source reflection coefficient so that a trade-off between noise figure and available gain can be made [1] and conjugate matching can be used at the output [2]. Available gain circles and noise figure circles described on the $\Gamma_{\mathrm{s}}$ plane are used to aid the selection of the soutce reflection coefficient [1]. Safety margins between the chosen values of source and toad reflection coefficients and their respective stability circles are included to allow for manufacturing variations [3][4]. Aithough the resulting amplifier is matched at its output port, the mismatch at the input port is typically severe and necessitates the trade-off of output match for improved input match. Circles of constant mismatch or VSWR [1] can be described on the load reflection coefficjent plane to aid this trade-off. The attractiveness of these graphical design mothods is that the functional hehaviour of gain, noise figure and mismatch with respect to the source and load reflection coefficients is obtained by simply plotting citcles whose centres and radii need only be calculated. A draw-back of this approach is that the selection of the source and load reflection coefficients is subjective, particularly when the contours of more than two performance paramcters are plotted on the same reflection coefficient plane. "Trial and error" is often required to obtain acceptable performance, and inevitably, circuit optimisers are required.

Other graphical methods have also been proposed recently [5] - [7] to aid the design procedure. Poole \& Paul [5], develop expressions for constant noise measure circles which can be used to design low noise amplifiers and considers both noise and gain simultancously without having to separately plot noise and available gain circles. Albirsson [6] investigated the mapping of noise circles onto the load plane to result in an LNA with matched input. Link \& Gudimetla [7] provide explicit design formulae for low noise amplifiers but do not consider stability.

In this paper, we propose a different approach whereby the functional behaviour of noise measure and port mismatch are described as functions of the stability safety margins rather than direct functions of the source and load reflection coefficients. As we shall see, this approach results in improved optimisation without requiring sophisticated circuit optimisers. Further, as the stability safety margins are used as design parameters, stability is directly considered without having to separately plot stability circles. In this work, we will asstme that the magnitudes of the transistor S-parameters $S_{11}$ and $S_{22}$ are both less than unity and that the stability circles do not enclose the origin. These assumptions are valid for many microwave transistors used in amplifier circuits. 


\section{Definitions}

In the work that follows, the definitions of the various reflection coefficients refer to the single-stage amplifier depicted in Fig. $l$. The transistor is cescribed by its $\mathbf{S -}$ parameters and noise parameters. The effective source and load reflection coefficients presented to the input and output ports of the transistor are $\Gamma_{S}$ and $\Gamma_{L}$ respectively. $\Gamma_{S}$ and $\Gamma_{L}$. typically result from transforming the extcrnal $50 \Omega$ source and load impedances via lossless networks. $\Gamma_{1}$ is the reflection coefficient looking into the input port of the transistor and is a function of $\Gamma_{\mathrm{L}}$ via the well known bilinear transformation [1]. Similarly $\Gamma_{2}$ is the reflection coefficient looking into the output port of the transistor and is a function of $\Gamma_{\mathrm{s}}$ via the well known bilinear trausformation [1].

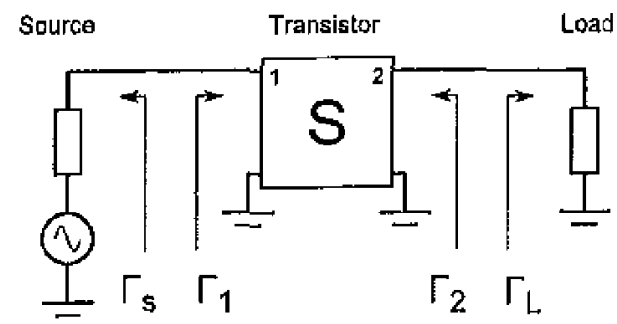

Fig. 1. Microwave amplifier circuit. $M_{1}$ :

In general, $\Gamma_{\mathrm{s}}$ and $\Gamma_{1}$ are mismatcled with mismatcl factor

$$
M_{1}=\frac{\left(1-\left|\Gamma_{s}\right|^{2}\right)\left(1-\left|\Gamma_{1}\right|^{2}\right)}{\left|1-\Gamma_{\mathrm{s}} \Gamma_{1}\right|^{2}}
$$

and $\Gamma_{\mathrm{L}}$ and $\Gamma_{2}$ are mismatched with mismatch factor $M_{2}$ :

$$
M_{2}=\frac{\left(1-\left|\Gamma_{\mathrm{L}}\right|^{2}\right)\left(1-\left|\Gamma_{2}\right|^{2}\right)}{\left|1-\Gamma_{\mathrm{L}} \Gamma_{2}\right|^{2}}
$$

The available gain $G_{A}$ and noise figure $F$ are functions of $\Gamma_{S}$ via the woll known formulae [1]. The centres and radii of the stability circles, and circles of constant available gain, noise figure, and mismatch are also given by well known formulae [1]. These circles are plotted so that the functional behaviour of the circuit with respect to $\Gamma_{\mathrm{S}}$ and $\Gamma_{\mathrm{L}}$ may be ascertained. It is of use to consider the noise measure which incorporates both $G_{A}$ and $F$ :

$$
N M=\frac{\not F-1}{1-\frac{1}{G_{d}}}
$$

The centres and radii of constant noise measure are available [5] but are generally umwieldy.

In the presence of statistical variation of both transistor and circuit parameters, it is important when sclecting $\Gamma_{S}$ and $\Gamma_{L}$ that an adequate safety margin with respect to their respective stability circies be maintained. Eccleston [3][4] has previously developed equations for maximum gain design using conditionally stable transistors and defined a new performance parameter, the stability safety margin. Let $C_{s}$ and $r_{s}$ be the centre and radius of the source stability circle, and $C_{t}$ and $r_{L}$ be the centre and radius of the lond stability circle. The source safety margin ( $S S M)$ is the distance between the nominal (and allowable) value of $\Gamma_{s}$ and the source stability circle. Likewise, the load safety margin $(L S M)$ is the distance between the rominal (and allowable) value of $\Gamma_{\mathrm{L}}$ and the load stability circle. A contour of constant $S S M$ is a circle with centre $C_{s}$ and radius $r_{S}+S S M$ when the origin is stable and outside the source stability circle [4]. Similarly for contours of constant $L S M$.

\section{Development}

In the approach that we propose the finctional behaviour of noise measure and port mismatch are described as finctions of the stability safety margins rather than direct functions of $\Gamma_{S}$ and $\Gamma_{\mathrm{L}}$. Since we assume that the transistor S-parameters $S_{11}$ and $S_{22}$ are both less than unity and that the stability circles do not enclose the origin, we can write $I_{S}$ and $\Gamma_{L}$ in terms of stability circle centres and radii, SSM and LSM:

$$
\begin{aligned}
& \Gamma_{\mathrm{S}}=C_{s}+\left(r_{s}+\operatorname{SSM}\right) \exp (j \theta) \\
& \Gamma_{\mathrm{L}}=C_{i}+\left(r_{i}+L S M\right) \exp (j \phi)
\end{aligned}
$$

where $\theta$ and $\phi$ are additional design parameters. With $\Gamma_{\mathrm{s}}$ and $\Gamma_{\mathrm{L}}$ described in this manner, $G_{A}$ and $F$ can be described as functions of $S S M$ and $\theta$. For a given value $\Gamma_{\mathrm{S}}, M_{1}$ and $M_{2}$ can be described as functions of $L S M$ and $\phi$. It is evident that $S S M$ and $L S M$ are design parameters as well as performance parameters.

To further simplify matters, two objective functions can be described. One of these being the noise measure and is described as a function of SSM and $\theta$ :

$$
F_{1}(S S M, \theta)=\frac{F(S S M, \theta)-1}{1-\frac{1}{G_{A}(S S M, \theta)}}
$$

The other being the product of $M_{1}$ and $M_{2}$ and is a function $L S M$ and $\phi$, for a given value of $\Gamma_{\mathrm{s}}$ : 


$$
F_{2}(L S M, \phi)=M_{1}\left[\Gamma_{\hat{i}}, L S M, \phi\right] M_{\hat{2}}\left[\Gamma_{2}\left(\Gamma_{g}\right), L S M, \phi\right]
$$

where $\mathrm{I}_{\mathrm{s}}$ is given by (4) alter SSM and $\theta$ have been detemined using (6). The expressions for $G_{A}, F, M_{1}$ and $M_{2}$ are well known and can be found in various texts [1]. Clearly we nced to minimise $F_{1}$ and maximise $F_{2}$ for the constraint that $S S M$ and $L S M$ are both greater than zero (or required safety margin), and the magnitudes of the resulting $\Gamma_{\mathrm{S}}$ and $\Gamma_{\mathrm{L}}$ are both less than unity.

\section{Numerical Example}

To illustrate the application of (4) to (7), we car consider an exanple of an LNA design that employs a transistor whose $\mathrm{S}$. parameters and noise parameters are:

$$
\begin{array}{ll}
S_{n t}=0.809 \angle \underline{-148^{\circ}} & S_{12}=0.111 /-4^{\circ} \\
S_{31}=1.940 \angle \underline{57^{\circ}} & S_{22}=0.634 \angle-48^{\circ} \\
\Gamma_{\text {Sopt }}=0.50 \angle \underline{130^{\circ}} & F_{\text {min }}=0.97 \mathrm{~dB} \\
R_{N}=2 \Omega &
\end{array}
$$

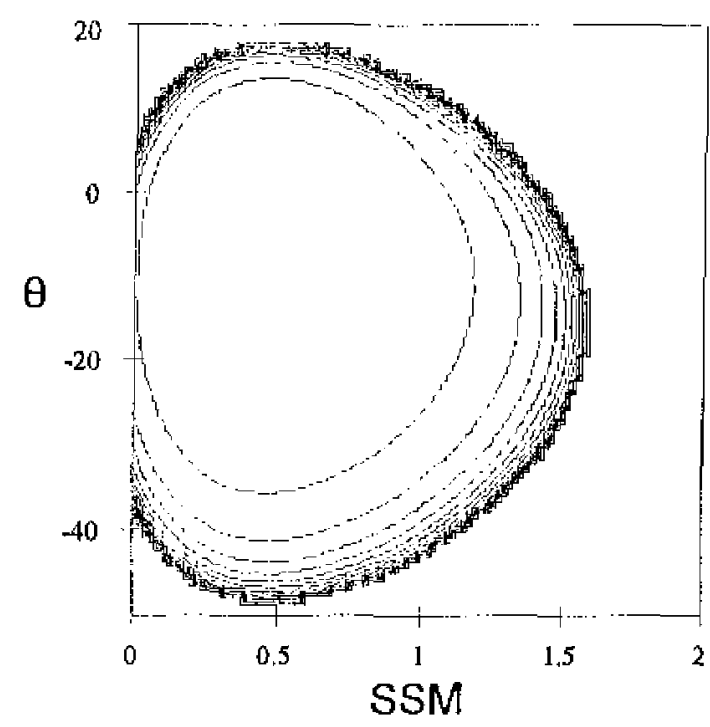

Fig 2. $F_{1}$ as a function of SSM and $\theta$ (deg).

Fig, 2 shows a plot of $F_{1}$ as a function of $S S M$ and $\theta$ (for $\left|\Gamma_{\mathrm{s}}\right|<1$ ). The ninimum of $\Gamma_{1}$ was located by a direct search of the $\operatorname{SSM}, \theta$ plane with a grid size of 0.02 in the $S S M$ direction and $0.7^{\circ}$ in the $\theta$ rlirection. The minimum of $F_{1}$ was found to be located at SSM equal to 0.46 and $\theta$ equal to $-4.5^{\circ}$ and results in $\Gamma_{\mathrm{s}}$ being equal to $0.52 \not 136^{\circ}, G_{A}$ equal to $10.3 \mathrm{~dB}$ and $F$ equal to $0.97 \mathrm{~dB}$.

With $\Gamma_{\mathrm{s}}$ equal to $0.52 \angle 136^{\circ}$, conjugate matching can be used at the output and results in an LSM of 0.33; however the input mismatch is 0.571 . ie only $57.1 \%$ of the available power from the input generator is delivered to the input of the transistor. We can however, plot $F_{2}$ as a function of $L S M$ and $\phi$ with $\Gamma_{\mathrm{s}}$ equal to 0.52 L136 as depicted in Fig. 3. The maximum of $F_{2}$ was located by a direct search of the $L S M, \phi$ plane with a grid size of 0.02 in the $L S M$ direction and $0.05^{\circ}$ in the $\phi$ direction. The maximum of $F_{2}$ was found to be located at $L S M$ equal to 0.54 and $\phi$ equal to $-84.6^{\circ}$ and results in $\Gamma_{\mathrm{L}}$ being equal to $0.47 / 35.9^{\circ}$. Under this condition, $M_{1}$ and $M_{2}$ are 0.786 and 0.903 respectively and has resulted in a $1.39 \mathrm{~dB}$ improvenent in the input match at the expense of only $0.44 \mathrm{~dB}$ ceterioration in the output matclt.

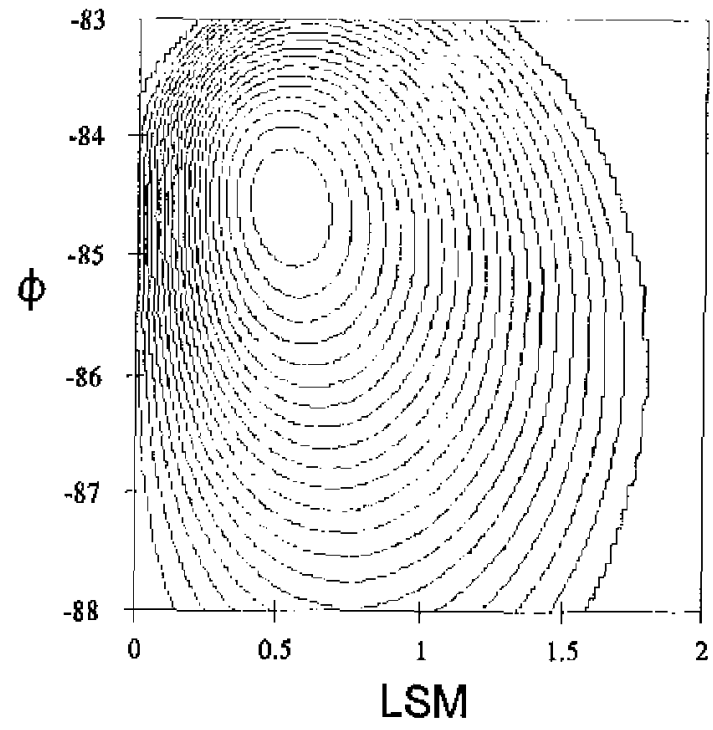

Fig. 3. $F_{2}$ as a function of $L S M$ and $\phi$ (deg).

Figs. 2 and 3 clearly show that the behaviour of $F_{1}$ and $F_{z}$ allows their minimisation and maximisation respectively to be achieved without resorting to sophisticated optimisers. Indeed, location of the minimum and maximum of $F_{1}$ and $F_{2}$ respectively only took a few seconds on a Pentium II. Since $S S M$ and $L S M$ are design parameters, stability margin is automatically included in the design process without plotting stability circles. So long as SSM and LSM are greater than 
zero, then $\Gamma_{\mathrm{S}}$ and $\Gamma_{\mathrm{L}}$ respectively are within the stable their segions.

We could have subjected the optimisation to constraints on $S S M$ and $L S M$, of which Figs. 2 and 3 indicate the ease with which this contd bave been done. In this case, one would only need to perform a one-dinuensional search for $\theta$ and $\phi$.

One will observe looking at Figs. 2 and 3 that the ranges of $\theta$ and $\phi$ are both narrow. The reason stems from the fact that these angles are with respect to the stability circle centres. Only small ranges of $\theta$ and $\phi$ are necessary to sweep the entire unit circle. This range decreases with increasing magnitudes of stability circle centres.

\section{Conclusion}

In this paper we have shown a method for selecting the values of the source and load reflection coefficients to achieve minimurn noise measure, and optimum isput and output match. This method is based on objective functions that are described in terms of stability safety margins, thereby incorporating stability into the design parameters rather than consider them separately. The objective functions are straightforwatd to optimise without sophisticated optimisers.

Work is continuing to extend this design approach to more generality as well as efficient means to locate the optimum values $S S M, L S M, \theta$ and $\phi$.

\section{References:}

[1] G. Gonzalez, Microwave Transistor Amplifers, Analysis and Design, Prentice-Hall 1997.

[2] M. L. Edwards, S. Cheng \& J. H Sinsky, "A Deterministic Approach for Designing Conditionally Stable Amplifiers", IEEE Transactions on Microwave Theory and Techniques, 41(2), July 1995, pp 1567. 1575 .

[3] K. W. Eccleston, "Design Formalae for Microwave Amplifiers Employing Conditionally-Stable FETs", 1998 Asia-Pacific Microwave Conference, Yokobama, Japan, 8-11 December 1998, pp 95 - 98.

[4] K. W. Eccleston, "Design Formulae for Microwave Amplifiers Employing Conditionally-Stable FETs", Accepted for publication in IElCE Trans, on Electronics, E82-C(7), July 1999.
[5] C. R. Poole \& D. K. Patl, "Optimum Noise Meastre Terminations for Microwave Transistor Amplifiers", IELE Transactions on Microwave Theory and Techniques, 33(11), November 1985, pp 1254-1257.

[6] B. M. Albinsson, "A Graplic Design Mcthod for Matched Low-Noise Amplifiers, IEEE Transactions on Microwave Theory and Tecliniques, 38(2), Febriary 1990, pp I18-122.

[7] G. N. Link \& V. S. Gudimetla, "Analytical Expressions for Simplifying the Design of Broadband Low Noise Microwave Amplifiers", IEEE Transactions. on Microwave Theory and Techniques, $43(10)$, October 1995, pp $2498-2501$. 